\title{
Unconventional magnetic properties of the icosahedral symmetry antiferromagnetic Heisenberg model
}

\author{
N. P. Konstantinidis* \\ Ames Laboratory and Department of Physics and Astronomy, Iowa State University, Ames, Iowa 50011, USA \\ (Received 20 November 2006; revised manuscript received 16 May 2007; published 27 September 2007)

\begin{abstract}
The antiferromagnetic Heisenberg model on icosahedral symmetry $I_{h}$ fullerene clusters exhibits unconventional magnetic properties despite the lack of anisotropic interactions. At the classical level, and for number of sites $n \leqslant 720$, the magnetization has two discontinuities in an external magnetic field, except from the dodecahedron where it has three, emphasizing the role of frustration introduced by the pentagons in the unusual magnetic properties. For spin magnitude $s_{i}=\frac{1}{2}$, there is a discontinuity of quantum character close to saturation for $n \leqslant 80$. This common magnetic behavior indicates that it is a generic feature of $I_{h}$ fullerene clusters, irrespective of $n$.
\end{abstract}

DOI: $10.1103 /$ PhysRevB.76.104434

PACS number(s): 75.10.Jm, 75.50.Ee, 75.50.Xx

\section{INTRODUCTION}

The antiferromagnetic Heisenberg model (AHM) is a prototype for strongly correlated electronic behavior, and the combination of low dimensionality, quantum fluctuations, and frustration produces unconventional magnetic behavior. ${ }^{1}$ This includes nonmagnetic excitations in the low-energy spectrum, nontrivial dependence of the specific heat and the susceptibility on temperature, and magnetization plateaux and discontinuities in a magnetic field. ${ }^{2-5}$ Here, the model is investigated for spins sitting on vertices of clusters of the fullerene type, with icosahedral spatial symmetry $I_{h}{ }^{6}$ Fullerene molecules have been found to superconduct when doped with alkali metals. ${ }^{7,8}$ An electronic mechanism for superconductivity was suggested based on perturbation theory calculations on the one-band Hubbard model on doped $C_{60}$, the fullerene with 60 carbon atoms and $I_{h}$ point group symmetry, which geometrically corresponds to the truncated icosahedron. ${ }^{9}$ Diagonalization of the Hubbard model is prohibitive due to limitations imposed by the dimensionality of the Hilbert space of this molecule. As a first step, the model is considered on its strong on-site repulsion limit at halffilling, the AHM, on clusters of $I_{h}$ symmetry with number of sites $n$ up to 720 . We look for correlations between the magnetic properties and spatial symmetry at the classical and quantum levels. The presence of such correlations could open the possibility of studying smaller clusters to gain insight on larger ones of the same symmetry, which are intractable with present day computational means. This approach could as well be used for the Hubbard model to investigate superconducting correlations.

Fullerene molecules are threefold coordinated and consist of 12 pentagons and $\frac{n}{2}-10$ hexagons. For $I_{h}$ symmetry, the number of sites is given by $n_{1}=20 i^{2}$ (to be called type I molecules), or $n_{2}=60 i^{2}$ (type II), with $i$ an integer. ${ }^{10,11}$ These clusters belong to the class of Goldberg polyhedra. The smallest is the dodecahedron with $n=20$ and no hexagons, and the largest considered here has $n=720$. Frustration is introduced by the pentagons, with each surrounded only by hexagons for $n>20$. The dodecahedron is the only cluster where pentagons are neighboring each other, hence frustration is maximal. As the number of hexagons increases with the number of sites, frustration on the average decreases. It has already been shown that there are strong similarities in the low-energy spectra, the specific heat, and the magnetic susceptibility of the dodecahedron and the icosahedron, a fivefold coordinated cluster with the same spatial symmetry and 12 sites consisting only of triangles. ${ }^{12}$ Here, the investigation is extended to the larger molecules, and show that spatial symmetry determines, to a large extent, the behavior in a magnetic field. We conclude that significant insight can be gained on more complicated fermionic models on large $I_{h}$ fullerene molecules from their solution on smaller molecules of the same symmetry.

The AHM is isotropic in spin space, and the total magnetization is usually a smooth function of an external magnetic field at the classical level for unfrustrated systems. Coffey and Trugman showed that it has a discontinuity for the dodecahedron and the truncated icosahedron $(n=60) .{ }^{13} \mathrm{It}$ was also shown that for individual spin magnitudes $s_{i}=\frac{1}{2}$ and 1 , the magnetization curve is discontinuous and the total spin changes by $\Delta S=4 s_{i}$ for the dodecahedron, twice as much as the change between adjacent $S$ sectors, with a particular sector never including the ground state in the field. ${ }^{12}$ The icosahedron also has a magnetization jump for classical spins and for higher $s_{i}{ }^{14}$ The icosidodecahedron is another molecule with $I_{h}$ symmetry, fourfold coordinated, and consisting of triangles and pentagons, with a similar property at high magnetic fields for the lowest $s_{i}{ }^{15}$ Here, the magnetic response of the AHM is calculated at the classical limit, $s_{i} \rightarrow \infty$, and the full quantum limit, $s_{i}=\frac{1}{2}$. We find that the response to an external magnetic field is discontinuous for all the $I_{h}$ fullerene clusters, showing the correlation between magnetic behavior and spatial symmetry. For classical spins, there are two discontinuities, one at relatively small magnetic fields and the second at high fields close to saturation. For the dodecahedron, another discontinuity precedes the low-field one, bringing the total number to 3 , a rather uncommon feature in the absence of anisotropic magnetic interactions. At the opposite limit, $s_{i}=\frac{1}{2}$, there is a jump with $\Delta S=2$ for the three smallest clusters $(n \leqslant 80)$, where the lowest energy state of the sector with five flipped spins from saturation and $S=\frac{n}{2}-5$ is never the ground state in a field. The mechanism of the jump is the same in all cases. For higher $n$ 's, memory 
requirements prohibit the calculation of the magnetization curve down to the appropriate fields. These common properties of the $I_{h}$ fullerenes point out the importance of symmetry and frustration for the determination of magnetic behavior.

The plan of this paper is as follows: In Sec. II, the model and the methods of solution are introduced, and in Secs. III and IV, the classical and $s_{i}=\frac{1}{2}$ cases are analyzed, respectively. Finally, Sec. V presents the conclusions.

\section{MODEL AND METHOD}

The antiferromagnetic Heisenberg Hamiltonian for spins $\vec{s}_{i}$ on the vertices $i$ of the clusters is

$$
H=J \sum_{\langle i, j\rangle} \vec{s}_{i} \cdot \vec{s}_{j}-h S^{z}
$$

where \langle\rangle denotes nearest neighbors, and $J$ is positive and is set equal to 1 , defining the unit of energy. $h$ is the strength of an external magnetic field in units of energy, and $S^{z}$ the projection of the total spin along the field direction $z$.

For classical spins, $\left|\vec{s}_{i}\right|$ is taken equal to 1 , and the energy is minimized as a function of the spin polar and azimuthal angles. ${ }^{13}$ For $s_{i}=\frac{1}{2}$, the Hamiltonian is block diagonalized according to its spatial and spin inversion symmetries, and a significant reduction in memory requirements is achieved. ${ }^{12,16-18}$

\section{CLASSICAL LIMIT}

In the absence of a field and type II clusters, the nearest neighbor pentagon correlations are equal to their isolated pentagon value $\cos \frac{4 \pi}{5}$, while nearest neighbor hexagon spins are antiparallel to each other. In other words, assembling the clusters from the individual polygons does not introduce further frustration. This is not the case for clusters of type I, with the pentagon and hexagon nearest neighbor correlations larger than $\cos \frac{4 \pi}{5}$ and -1 , respectively. As the number of sites increases though, and hexagons strongly dominate in number, pentagon correlations approach their isolated pentagon values, while the rest of the bonds become more and more antiparallel. The bonds that approach being antiparallel the fastest are the ones between spins belonging to pentagons and their hexagon nearest neighbors. Figure 1 shows how the energy per site decreases with the number of sites and approaches the unfrustrated hexagon-only limit of $-\frac{3}{2}$.

The saturation fields $h_{\text {sat }}$ for the icosahedral clusters are given in Table I. They are an increasing function of the number of sites, and are converging to the value of the honeycomb lattice, $h_{\text {sat }}=6$. In contrast to the lattices considered by Zhitomirsky et al. in Ref. 19 the polygons of the fullerene clusters are edge rather than corner sharing and of two different kinds, and as a result, the Hamiltonian cannot be written in the form of a sum of the squared total spin of individual units. Thus numerical values are presented for the saturation fields in Table I, except for the dodecahedron and the truncated icosahedron where analytic expressions are available. For these two clusters, the expression of the energy for high fields is simple enough to yield the saturation

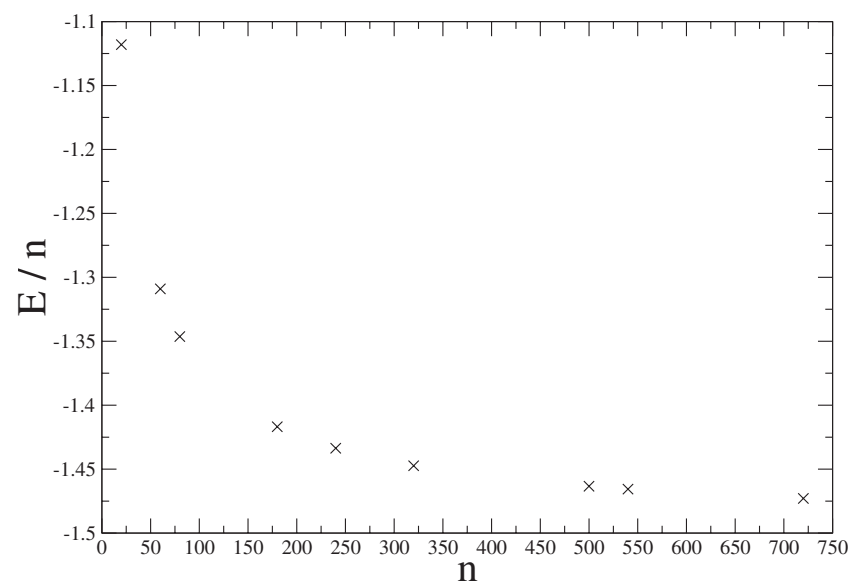

FIG. 1. Classical energy per site $\frac{E}{n}$ as a function of the number of sites $n$. The unfrustrated hexagon-only value is $-\frac{3}{2}$.

field in analytic form (Ref. 20 presents the high-field energy functional for the dodecahedron).

The $I_{h}$ clusters exhibit two magnetization discontinuities in an external field, with the critical values listed in Table I. Varying the magnetic field with a very fine step captures all of them, some of which were missed for the clusters studied in Ref. 13, the dodecahedron and the truncated icosahedron. Typical plots are shown in Figs. 2 and 3 for $n=540$ and 720, a type II cluster and a type I cluster, respectively. As the field is increased from zero, the spins gradually turn toward its direction. The number of different polar angles of the spin configuration can be expressed in terms of the integer $i$ that gives the number of sites, $n_{1}$ and $n_{2}$. For the low-field phase, to be called I from now on, there are $4 i$ distinct polar angles, each corresponding to a group of five spins, plus $2 i(i-1)$ more, each representing ten spins, for clusters of type I. The spins of each of the $4 i$ groups are equally distributed in the azimuthal plane with a step equal to $\frac{2 \pi}{5}$. Half of the groups have exactly the same $\phi$ 's, and similarly for the other half of the groups. These two families differ in azimuthal angles by $\frac{\pi}{5}$. They define a set of ten lines in the $x y$ plane with respect to which the spins are symmetrically placed. For type II clusters, there are $6 i^{2}$ distinct polar angles, each corresponding to a group of ten spins. Again the spins in each group are equally distributed in the azimuthal plane with a step of $\frac{2 \pi}{5}$, with each $\phi$ value shared by two spins. The phase above the first critical field will be called phase II, and has $\frac{n}{4}$ different polar angles, each including four spins for both types of clusters. All the spins now have negative magnetic energy. There are two perpendicular lines in the azimuthal plane, with the spins symmetrically placed with respect to both. For higher fields, the clusters enter phase III, the most symmetric phase. For clusters of type I, this phase is derived from phase I by further increasing the symmetry by a factor of 2 . The number of distinct polar angles is $2 i$, each including ten spins, plus $i(i-1)$, each having 20 spins. The $2 i$ groups of ten spins have exactly the same set of azimuthal angles, distributed evenly around the $z$ axis with a step of $\frac{\pi}{5}$. These spins define a set of five lines, which, along with the ones that bisect them, have the spins symmetrically placed around them in the azimuthal 
TABLE I. Magnetic fields with a classical magnetization discontinuity $h_{c}$ over the saturation field $h_{\text {sat }}$ for $n$ sites, and reduced magnetization values on the sides of the discontinuity $M_{-} / n$ and $M_{+} / n$. For the dodecahedron $(n=20)$, the numbers in the left part of the table correspond to the phase Ia to phase II transition. The corresponding numbers for the phase I to phase Ia transition are $0.26350,0.22411$, and 0.22660 . The last column shows the saturation field value.

\begin{tabular}{|c|c|c|c|c|c|c|c|}
\hline \multirow[b]{2}{*}{$n$} & \multicolumn{3}{|c|}{ Phase $\mathrm{I} \rightarrow \mathrm{II}$} & \multicolumn{3}{|c|}{ Phase $\mathrm{II} \rightarrow$ III } & \multirow[b]{2}{*}{$h_{\text {sat }}$} \\
\hline & $\frac{h_{c}}{h_{\text {sat }}}$ & $\frac{M_{-}}{n}$ & $\frac{M_{+}}{n}$ & $\frac{h_{c}}{h_{s a t}}$ & $\frac{M_{-}}{n}$ & $\frac{M_{+}}{n}$ & \\
\hline 20 & 0.26983 & 0.23688 & 0.27518 & 0.73428 & 0.74766 & 0.75079 & $3+\sqrt{5}$ \\
\hline 60 & 0.14692 & 0.11723 & 0.14790 & 0.94165 & 0.94574 & 0.94651 & $\frac{9+\sqrt{5}}{2}$ \\
\hline 80 & 0.12596 & 0.10003 & 0.12702 & 0.95134 & 0.95465 & 0.95527 & 5.69932 \\
\hline 180 & 0.08275 & 0.06438 & 0.08325 & 0.98013 & 0.98208 & 0.98234 & 5.84925 \\
\hline 240 & 0.07139 & 0.05525 & 0.07176 & 0.98541 & 0.98697 & 0.98716 & 5.88350 \\
\hline 320 & 0.06161 & 0.04749 & 0.06190 & 0.98902 & 0.99027 & 0.99041 & 5.91056 \\
\hline 500 & 0.04910 & 0.03765 & 0.04928 & 0.99301 & 0.99386 & 0.99396 & 5.94104 \\
\hline 540 & 0.04722 & 0.03618 & 0.04738 & 0.99355 & 0.99434 & 0.99443 & 5.94517 \\
\hline 720 & 0.04082 & 0.03120 & 0.04094 & 0.99516 & 0.99577 & 0.99584 & 5.95829 \\
\hline
\end{tabular}

plane. For clusters of type II, there are $2 i$ different polar angle values, each corresponding to ten spins, plus $(3 i-1) i$ values, each representing 20 spins. Each azimuthal angle is shared by two spins in the same group. The azimuthal angles of the groups of ten spins are equally distributed with a step of $\frac{2 \pi}{5}$, and define symmetry lines in the $x y$ plane.

For the dodecahedron, the only member of the icosahedral family that consists exclusively of pentagons, there is one more phase that minimizes the energy in a very narrow window between phases I and II. This will be called Ia, and the spins have distinct polar angles in a plane that bisects the cluster and contains four of them. Their azimuthal angles are equal in pairs, with the two values differing by $\pi$. The rest of

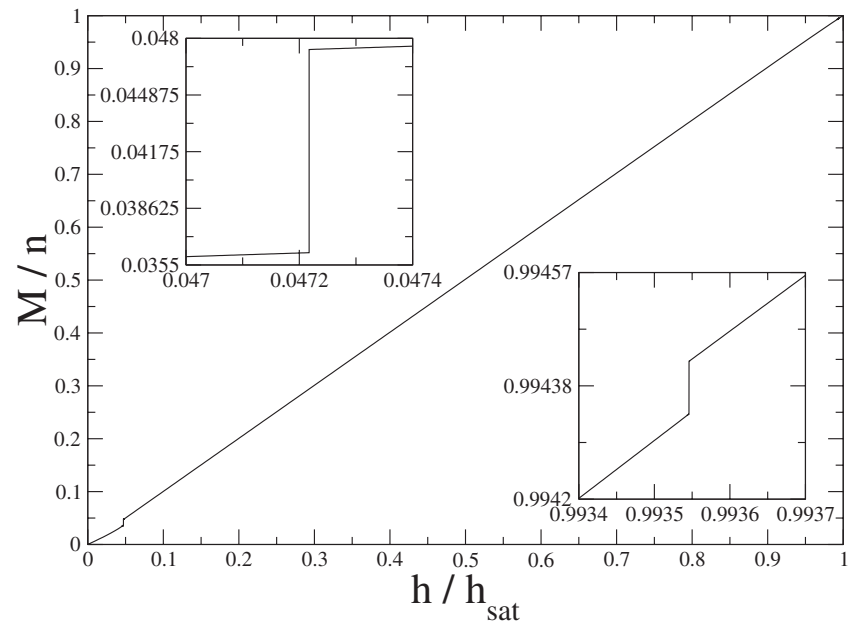

FIG. 2. Reduced magnetization $\frac{M}{n}$ for a number of sites $n$ $=540$, as a function of the magnetic field $h$ over its saturation value $h_{\text {sat }}$. The insets show the details of the low- and high-field magnetization discontinuities, which occur at magnetic fields $h_{c}$ such that $\frac{h_{c}}{h_{\text {sat }}}=0.04722$ and 0.99355 respectively. the spins have common polar angles in pairs, symmetrically placed around the plane that contains these four spins, while their azimuthal angles are also symmetrically spaced with respect to the azimuthal direction of the four plane spins. ${ }^{20}$

Another way to characterize the symmetry of the various phases is with the symmetry operations of the $I_{h}$ group that do not change the polar angle and the relative azimuthal angles with the other spins for every spin. These operations form a subgroup of $I_{h}$. For phase I, it is $C_{5 v}$ for clusters of type I, and $S_{10}$ for clusters of type II. For phase II, the subgroup is $D_{2}$, and for phase III, it is $D_{5 d}$, irrespective of the type of the cluster. In phase Ia of the dodecahedron, the symmetry group of the ground state is $C_{s}$.

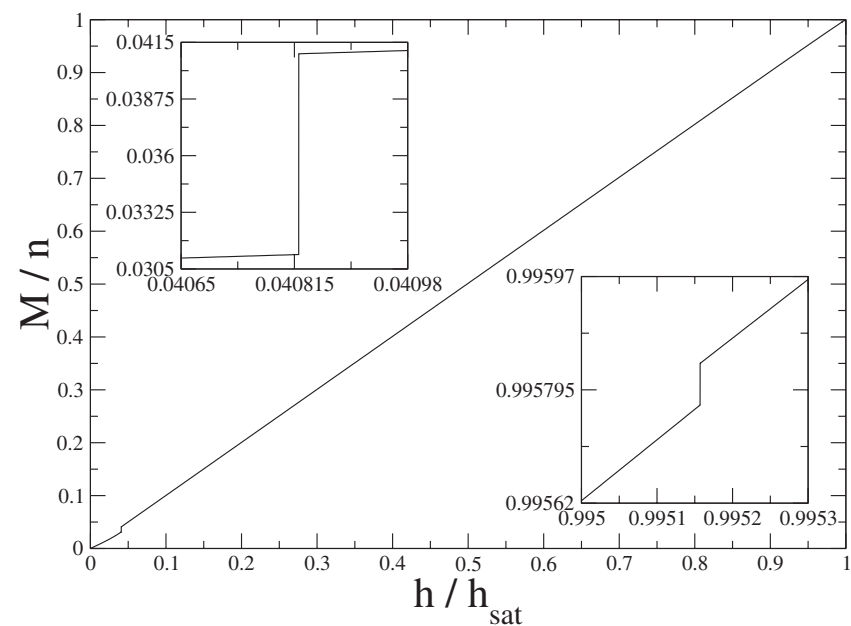

FIG. 3. Reduced magnetization $\frac{M}{n}$ for a number of sites $n$ $=720$, as a function of the magnetic field $h$ over its saturation value $h_{\text {sat }}$. The insets show the details of the low- and high-field magnetization discontinuities, which occur at magnetic fields $h_{c}$ such that $\frac{h_{c}}{h_{\text {sat }}}=0.04082$ and 0.99516 respectively. 


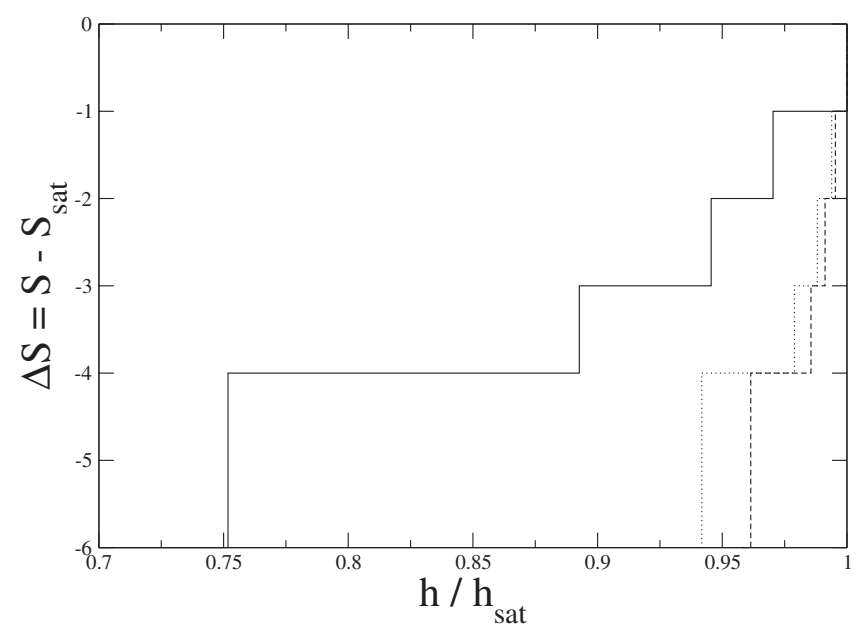

FIG. 4. Difference $\Delta S$ between the total spin in the ground state for $s_{i}=\frac{1}{2}, S$, and its saturated value $S_{\text {sat }}=\frac{n}{2}$, with $n$ the number of sites, as a function of the magnetic field $h$ over its saturation value $h_{\text {sat }}$. Solid line, $n=20$; dotted line, $n=60$; and dashed line, $n=80$. The discontinuities occur at magnetic fields $h_{c}$ such that $\frac{h_{c}}{h_{s a t}}$ $=0.75177,0.94179$, and 0.96142 , respectively.

Table I shows that as frustration decreases with $n$, phase II, the least symmetric around the $z$ axis, becomes the ground state for a wider range of fields. In contrast, phase I is suppressed to lower fields, and phase III appears just before saturation. The change in the magnetization $\Delta M$ over the saturation magnetization $n$ decreases with $n$ for both transitions. These results indicate that the discontinuities will occur for any value of $n$, albeit closer to $h=0$ and $h=h_{\text {sat }}$ and with smaller $\frac{\Delta M}{n}$ as $n$ increases. The role of the pentagons, which introduce frustration in the system, is not diminished even when the hexagons strongly prevail in number. It is also noted that hysteresis curves were calculated by slowly increasing the field from zero to saturation and then switching it off to zero in the same manner. In both cases, the magnetization curve is the same and no hysteresis is observed. All the clusters are in phase I for lower fields, and in phase III for higher fields, with a transition having discontinuous magnetic susceptibility between the two phases. ${ }^{20}$

$$
\text { IV. } s_{i}=\frac{1}{2}
$$

In the extreme quantum case $s_{i}=\frac{1}{2}$, the magnetization curve typically follows a step-like structure, with $\Delta S=1$ between adjacent $S$ sectors. However, frustration can lead to magnetization discontinuities. It has been found for the dodecahedron that the spin sector $S=\frac{n}{2}-5$ with five flipped spins from saturation never includes the ground state in a field, resulting in a step $\Delta S=2 .{ }^{12}$ For $s_{i}=1$, a similar discontinuity was found, along with a second one for lower $S$. The calculation is here extended to the truncated icosahedron and the $n=80$ cluster, where memory requirements permit Lanczos diagonalization for at least $S^{z}=\frac{n}{2}-6$. Similar to the dodecahedron, there is a magnetization discontinuity for both clusters with a step $\Delta S=2$ between sectors on the sides of $S=\frac{n}{2}-5$ (Fig. 4). The mechanism of the jump is the same in
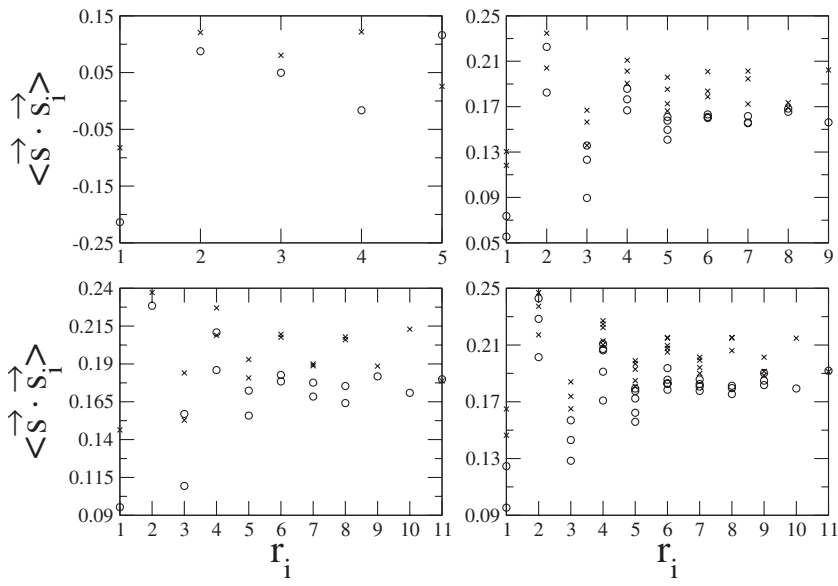

FIG. 5. Correlation functions $\left\langle\vec{s} \cdot \vec{s}_{i}\right\rangle$ between a spin $\vec{s}$ and the rest of the spins $\vec{s}_{i}$ for $s_{i}=\frac{1}{2}$ as a function of distance $r_{i}$ (the unit of distance is the distance between nearest neighbors, taken equal for all their pairs). $\bigcirc, S=\frac{n}{2}-6$ and $\times, S=\frac{n}{2}-4$ ( $S$ is the total spin, $n$ the number of sites). Upper left, $n=20$; upper right, $n=60$; lower left, $n=80$, site belonging only to hexagons; and lower right, $n=80$, site belonging to a pentagon.

all three cases. The ground state below and above the transition is nondegenerate. It switches from the $A_{g}$ to the $A_{u}$ one-dimensional irreducible representation of the $I_{h}$ symmetry group as the field increases, changing its spatial symmetry from symmetric to antisymmetric. ${ }^{6}$ For the dodecahedron, the magnetization curve has also been calculated for higher quantum numbers up to $s_{i}=\frac{5}{2}$ close to saturation, and there are no discontinuities related to the sector with five flipped spins for $s_{i}>1$. The same is true for the truncated icosahedron when $s_{i}=1$ or $\frac{3}{2}$. This indicates that the jump is not related to the classical limit, but rather is a purely quantum effect. Similar to the classical case, the discontinuities appear for fields $h_{c}$ closer and closer to the saturation field with increasing $n$, at $\frac{h_{c}}{h_{s a t}}=0.75177,0.94179$, and 0.96142 , respectively.

A magnetization discontinuity close to saturation has been found by Schulenburg et al. for various types of lattices. ${ }^{4}$ The authors were able to analytically show how the presence of localized magnons leads to it. The fullerene molecules have edge-sharing polygons of two different types, and such an analysis is not obvious. All the correlation functions $\left\langle\vec{s} \cdot \vec{s}_{i}\right\rangle$ between a spin $\vec{s}$ and the rest of the spins $\vec{s}_{i}$ have been calculated for the $n \leqslant 80$ clusters around the discontinuity, and are shown in Fig. 5. Their values are not simple fractions as in Ref. 4. On the average, they follow an antiferromagnetic pattern, increasing and decreasing alternatively as a function of distance, while for longer distances, this fluctuation pattern disappears. For the two type I clusters, the longest distance correlation function becomes more antiferromagnetic above the transition (dodecahedron), or does not change in character $(n=80)$. This is not true for the truncated icosahedron, indicating one more difference between clusters of types I and II.

\section{CONCLUSIONS}

The common magnetic properties of the AHM for all the $I_{h}$ fullerene clusters investigated for $s_{i} \rightarrow \infty$ and $s_{i}=\frac{1}{2}$ suggest 
that they are shared by all the clusters of this class, independent of $n$. Frustration, spatial symmetry, and the presence of pentagons result in magnetization discontinuities which are uncommon for a model lacking magnetic anisotropy. Combined with the similarities in the low-energy spectra and thermodynamic properties of $I_{h}$ clusters found in Ref. 12, the results presented here show that predictions for the behavior of fermionic models on $I_{h}$ clusters can be made by studying smaller clusters of the same symmetry. In particular, comparison of the energy of neutral $C_{60}$ plus two electrons to the total energy of two separate molecules of neutral $C_{60}$ where one electron has been added to each, shows if there is an effective attractive interaction between the two electrons, favoring superconductivity. This calculation is much more tractable in the Hilbert space of the dodecahedron than that of the truncated icosahedron..$^{21}$

In summary, the magnetization of the AHM for fullerene clusters of spatial symmetry $I_{h}$ has been shown to exhibit discontinuities in a field ranging up to 3 at the classical level. The results indicate that the discontinuities are a feature of any $I_{h}$ fullerene cluster, even though phase II is strongly predominant with increasing $n$. For $s_{i}=\frac{1}{2}$, there is also a jump for higher fields for $n \leqslant 80$, which is of purely quantum char- acter. Again it is anticipated that this is a generic feature of the AHM on fullerene molecules with $I_{h}$ symmetry. These effects are nontrivial in the absence of anisotropic magnetic terms from the Hamiltonian. The common spatial symmetry of the $I_{h}$ fullerenes leads to similar magnetic behavior. It is of interest to examine correlations between spatial symmetry and magnetic properties for other types of symmetry as well as investigate correlations between electronic behavior and spatial symmetry for more complicated models with orbital degrees of freedom. The findings of this paper show that insight on the superconducting properties of the truncated icosahedron can be gained from considering the significantly smaller Hilbert space of the dodecahedron.

\section{ACKNOWLEDGMENTS}

The author thanks D. Coffey for discussions and clarifications on various issues in this paper, and M. Luban, P. Herzig, and L. Engelhardt for discussions. Ames Laboratory is supported by the United States Department of EnergyBasic Energy Sciences under Contract No. DE-AC02$07 \mathrm{CH} 11358$.
*Present address: Institut für Festkörperforschung-Theorie III, Forschungszentrum Jülich, Leo-Brandt-Strasse, 52425 Jülich, Germany and Institut für Theoretische Physik A, Physikzentrum, RWTH Aachen, 52056 Aachen, Germany.

${ }^{1}$ G. Misguich and C. Lhuillier, in Frustrated Spin Systems, edited by H. T. Diep (World Scientific, Singapore, 2003).

${ }^{2}$ C. Waldtmann, H.-U. Everts, B. Bernu, C. Lhuillier, P. Sindzingre, P. Lecheminant, and L. Pierre, Eur. Phys. J. C 2, 501 (1998).

${ }^{3}$ N. P. Konstantinidis, arXiv:cond-mat/0503659 (unpublished).

${ }^{4}$ J. Schulenburg, A. Honecker, J. Schnack, J. Richter, and H.-J. Schmidt, Phys. Rev. Lett. 88, 167207 (2002).

${ }^{5}$ A. Honecker, J. Schulenburg, and J. Richter, J. Phys.: Condens. Matter 16, 749 (2004).

${ }^{6}$ S. L. Altmann and P. Herzig, Point-Group Theory Tables (Oxford University Press, New York, 1994).

${ }^{7}$ A. F. Hebard, M. J. Roseeinsky, R. C. Haddon, D. W. Murphy, S. H. Glarum, T. T. M. Palstra, A. P. Ramirez, and A. R. Kortan, Nature (London) 350, 600 (1991).

${ }^{8}$ K. Holczer, O. Klein, S.-M. Huang, R. B. Kaner, K.-J. Fu, R. L. Whetten, and F. Diederich, Science 252, 1154 (1991).
${ }^{9}$ S. Chakravarty, M. Gelfand, and S. Kivelson, Science 254, 970 (1991).

${ }^{10}$ M. Fujita, R. Saito, G. Dresselhaus, and M. S. Dresselhaus, Phys. Rev. B 45, 13834 (1992).

${ }^{11}$ M. Yoshida, http://cochem2.tutkie.tut.ac.jp:8000/Fuller/

${ }^{12}$ N. P. Konstantinidis, Phys. Rev. B 72, 064453 (2005).

${ }^{13}$ D. Coffey and S. A. Trugman, Phys. Rev. Lett. 69, 176 (1992).

${ }^{14}$ C. Schröder, H.-J. Schmidt, J. Schnack, and M. Luban, Phys. Rev. Lett. 94, 207203 (2005).

${ }^{15}$ R. Schmidt, J. Richter, and J. Schnack, J. Magn. Magn. Mater. 295, 164 (2005).

${ }^{16}$ B. Bernu, P. Lecheminant, C. Lhuillier, and L. Pierre, Phys. Rev. B 50, 10048 (1994).

${ }^{17}$ O. Waldmann, Phys. Rev. B 61, 6138 (2000).

${ }^{18}$ W. Florek and S. Bucikiewicz, Phys. Rev. B 66, 024411 (2002).

${ }^{19}$ M. E. Zhitomirsky, A. Honecker, and O. A. Petrenko, Phys. Rev. Lett. 85, 3269 (2000).

${ }^{20}$ N. P. Konstantinidis and M. Luban (unpublished).

${ }^{21}$ F. Lin, E. S. Sørensen, C. Kallin, and A. J. Berlinsky, Phys. Rev. B 76, 033414 (2007). 